ÉGYPTE

monde arabe

\section{Égypte/Monde arabe}

12-13 | 1993

Une économie en transition

\title{
Impact de la réforme économique sur les chances de scolarisation en Égypte
}

\section{Mona Mustafa Al-baradi'i}

\section{Q OpenEdition}

\section{Journals}

Édition électronique

URL : https://journals.openedition.org/ema/1266

DOI : 10.4000/ema.1266

ISSN : 2090-7273

\section{Éditeur}

CEDEJ - Centre d'études et de documentation économiques juridiques et sociales

\section{Édition imprimée}

Date de publication : 31 mars 1993

Pagination : 179-195

ISSN : 1110-5097

\section{Référence électronique}

Mona Mustafa Al-baradi'i, « Impact de la réforme économique sur les chances de scolarisation en

Égypte », Égypte/Monde arabe [En ligne], 12-13 | 1993, mis en ligne le 08 juillet 2008, consulté le 07 juillet 2022. URL : http://journals.openedition.org/ema/1266 ; DOI : https://doi.org/10.4000/ema.1266

Ce document a été généré automatiquement le 7 juillet 2022.

Tous droits réservés 


\title{
Impact de la réforme économique sur les chances de scolarisation en Égypte
}

\author{
Mona Mustafa Al-baradi'i
}

1 L'enseignement est un des défis essentiels de l'Égypte, eu égard aux contraintes qu'impose à ce secteur une politique de restriction budgétaire, d'une part, et aux exigences d'une réforme impliquant une amélioration qualitative de l'enseignement, d'autre part.

2 Dès après la Révolution de Juillet, l'Égypte a adopté une série de mesures visant à élargir l'accès à tous les cycles de l'enseignement, d'où une nette croissance du taux de scolarisation : le nombre d'élèves du cycle primaire est ainsi passé d'1 million en 1952 à environ 7 millions en 1989, soit un taux de croissance annuel moyen de 5,1\%. Dans le cycle secondaire, le nombre d'élèves, qui était de 154000 en 1952, a atteint 3,8 millions en 1989 , ce qui représente un taux de croissance moyen annuel de $9,1 \%$. Il est de 7,1\% dans le cycle supérieur, qui est passé de 37000 à 500000 étudiants environ au cours de la même période ${ }^{1}$.

3 La gratuité de l'enseignement à tous les niveaux - principe constitutionnel - a certes joué un rôle important dans l'égalisation des chances de scolarisation (selon certains auteurs, l'enseignement était, à l'époque nassérienne, un facteur puissant d'ascension sociale) ${ }^{2}$. Mais en dépit de cette vaste expansion, l'Égypte n'est pas encore parvenue à scolariser tous les enfants ni à enrayer totalement l'analphabétisme.

4 En effet, bien que le taux de scolarisation, dans le cycle primaire, soit passé de $41 \%$ en 1952 à $72 \%$ en $1975^{3}$, puis à $90 \%$ en $1986^{4}$, ce qui est sans doute très performant, il n'en demeure pas moins que l'on compte encore $10 \%$ d'enfants - 160.000 enfants environ, filles surtout- non scolarisés. Ce pourcentage, bien que relativement bas, est inquiétant pour deux raisons : d'une part, il concerne surtout les couches les plus défavorisées ${ }^{5}$; d'autre part, ce groupe sera le plus difficile à scolariser; des mesures particulières devront être adoptées, avec les frais considérables que cela implique ${ }^{6}$. 
Quant au nombre d'analphabètes, il demeure élevé : on en comptait 15,1 millions en $1976^{7}$ et, en 1986, 17,6 millions (soit plus de la moitié de la population de 10 ans et plus), soit un taux d'analphabétisme de 49,6\% (37,8 \% dans la population masculine, 61,8 \% dans la population féminine) ${ }^{8}$.

6 Jusqu'à quel point cette extension de l'accès à l'enseignement a-t-elle contribué à réaliser l'égalité des chances, dont la scolarisation gratuite était une première étape? Nous tenterons de répondre à cette question en étudiant, d'une part, l'offre de scolarisation, et, d'autre part, l'inégalité des chances du point de vue de la demande.

\section{L'offre de scolarisation}

7 Nous aborderons successivement :

1. la répartition des chances de scolarisation entre les différents gouvernorats (autrement dit, la répartition des établissements scolaires et le taux de réalisation de leurs objectifs quantitatifs, exprimés en pourcentages de scolarisation);

2. la répartition des chances de scolarisation entre garçons et filles ;

3. la répartition du budget de l'enseignement entre les différents gouvernorats, les différents cycles, les dépenses courantes et les investissements.

4. le volume de l'enseignement privé, comparativement à celui de l'enseignement public, et l'effet de cette répartition sur les chances de scolarisation.

\section{Répartition des chances de scolarisation entre les gouvernorats}

8 - Répartition des établissements scolaires entre les différents cycles et types d'enseignement (voir tableau 1)

9 Le nombre d'écoles publiques gratuites a atteint, en 1989/1990, 20.034 pour les trois cycles de l'enseignement, dont 13.953 écoles primaires (69,6\%), 5.059 écoles élémentaires $(25,3 \%)$ et 1.022 écoles secondaires $(5,1 \%)$.

Tableau 1 : Répartition des écoles gouvernementales selon les cycles et les types d'enseignement 1989-90

\begin{tabular}{|l|r|r|r|r|r|r|r|r|}
\hline Cycle & \multicolumn{2}{|c|}{ Officiel } & \multicolumn{2}{c|}{$\begin{array}{c}\text { Officiel } \\
\text { de langues }\end{array}$} & \multicolumn{2}{c|}{$\begin{array}{c}\text { Privé } \\
\text { Gratuit }\end{array}$} & \multicolumn{2}{c|}{ Total } \\
\hline & $\mathrm{Nb}$ & $\%$ & $\mathrm{Nb}$ & $\%$ & $\mathrm{Nb}$ & $\%$ & $\mathrm{Nb}$ & $\%$ \\
\cline { 2 - 9 } & 13.527 & 97,0 & 46 & 0,3 & 380 & 2,7 & 13.953 & 100 \\
Primaire & 4.918 & 97,2 & 37 & 0,8 & 104 & 2,0 & 5.059 & 100 \\
Elémentaire & 1.022 & 100 & - & - & - & - & 1.022 & 100 \\
Secondaire & & & & & & & & \\
\hline
\end{tabular}

Source : données du ministère de l'Éducation.

10 - Répartition des établissements scolaires entre les gouvernorats. La comparaison des répartitions, entre gouvernorats, des écoles primaires d'un côté et de la population âgée de 6 à 12 ans de l'autre, révèle de fortes disparités géographiques. Avec 11,7 \% de la population mais $6,2 \%$ des écoles, Le Caire est le plus désavantagé. 
11 La répartition des écoles élémentaires et secondaires se rapproche davantage de celle de la population concernée. Contrairement à la situation qui est la sienne pour les écoles primaires, le gouvernorat du Caire bénéficie d'un équilibre entre pourcentage d'écoles élémentaires et secondaires et taux de population (12-20 ans). Fait remarquable, la répartition écoles/population la plus déséquilibrée se concentre dans les gouvernorats de Haute-Égypte (Giza, Fayoum, Béni-Souef, Miniya, Assiout, Sohag et Qena).

12 - Répartition des taux de scolarisation entre les gouvernorats. Afin de mesurer l'inégalité des chances de scolarisation entre les différents gouvernorats, nous avons utilisé la courbe de Lorenz et le coefficient de Gini. La courbe de Lorenz (ou courbe de concentration) permet de comparer les pourcentages de scolarisation dans les différents gouvernorats ou tranches de population (les taux cumulés des élèves d'un cycle donné apparaissant sur l'ordonnée, et ceux de la population de l'âge concerné - correspondant au cycle d'enseignement mesuré sur l'ordonnée - apparaissant sur l'abscisse en ordre croissant).

13 La courbe de Lorenz indique une inégalité relativement modérée entre les différents gouvernorats, dont les taux s'étagent de 63,78 \% à 98,67 \% (il existe deux gouvernorats pour lesquels les taux de scolarisation sont supérieure à $100 \%$, Qaliubiyya et Alexandrie, ce qui traduit soit une sur-évaluation du nombre d'enfants scolarisés, soit une sous-évaluation du nombre d'enfants d'âge scolaire. Huit gouvernorats possèdent encore, par ordre croissant, des taux de scolarisation inférieurs à $75 \%$.

14 Le recours aux mêmes instruments (courbe de Lorenz et coefficient de Gini) pour mesurer la répartition de la scolarisation des enfants de 12 à 20 ans dans les cycles élémentaire et secondaire ${ }^{9}$ révèle une plus grande inégalité entre gouvernorats que pour le cycle primaire : le taux de scolarisation varie entre 29,88 dans le gouvemorat du Fayoum et 74,10 dans celui de Marsa-Matrouh (écart de 0,3860 selon le coefficient de Gini). Notons que les taux de scolarisation en Égypte (surtout dans le primaire) doivent être considérés avec prudence, étant donné la forte proportion d'abandon des études : $30 \%$ dans le cycle primaire pour la période allant de 1978 à 1985.

\section{Inégalité des sexes devant l'école}

Toutes les estimations révèlent une inégalité entre garçons et filles malgré l'amélioration survenue ces dernières années (tableau 2).

16 Si les pourcentages de garçons et de filles scolarisés sont comparables dans certains gouvernorats - Le Caire $(69,27 \%$ de garçons et $68,18 \%$ de filles) et Alexandrie (80,50 \% de garçons et $77,13 \%$ de filles) - l'écart garçons/filles demeure marqué dans les gouvernorats de Haute-Égypte (Giza, Fayoum, Béni-Souef, Minya, Assiout, Sohag et Qena). L'écart le plus grand se trouve dans le gouvemorat de Minya, où le taux de scolarisation des filles atteint 59 \% de celui des garçons. Le même écart apparaît en ce qui concerne le taux de scolarisation des garçons et des filles dans les cycles élémentaire et secondaire: là encore, ce sont les gouvernorats de Haute-Égypte qui montrent l'écart le plus grand entre les sexes. Au niveau national, la moyenne de scolarisation des garçons est de $86,16 \%$, contre $74,55 \%$ pour les filles. Pour les deux cycles élémentaire et secondaire, la proportion est de $44,28 \%$ et de $39,02 \%$ respectivement. 
Tableau 2 : Pourcentage de filles dans les différents cycles de renseignement

\begin{tabular}{|l|c|c|c|c|}
\hline & $1972-73$ & $1981-82$ & $1983-84$ & $1985-86$ \\
\hline & & & & \\
Primaire & 38,0 & 40,9 & 42,2 & 44,1 \\
Préparatoire & 33,0 & 38,5 & 39,4 & 40,3 \\
Secondaire & 32,1 & 36,7 & 37,0 & 37,6 \\
Universitaire & 29,9 & 32,0 & 34,6 & 35,0 \\
\hline
\end{tabular}

Source : World Bank, Poverty alleviation and adjustment in Egypt.

Report n 8515. EGT, volume II. Main report, June 6, 1990. Table 3. 10. p. 13.

17 Néanmoins, les chiffres concernant les gouvemorats de Gharbiyya et de Marsa-Matrouh seront considérés avec prudence.

Remarquons que cette répartition inégalitaire des taux de scolarisation garçons/filles a des répercussions négatives dans maints domaines (santé, alimentation, environnement, productivité, planning familial, etc.). Le ministère de l'Éducation (et d'autres avec lui) devra donc déployer davantage d'efforts pour élever le taux de scolarisation des filles. Il ne s'agit pas seulement de construire davantage d'écoles, mais de prendre en compte les facteurs entrant dans la demande individuelle de scolarisation, ce qui augmentera nettement le coût des dépenses d'enseignement.

\section{Répartition du budget de l'enseignement}

\section{Répartition des dépenses entre les différents gouvernorats}

La répartition des dépenses d'enseignement par gouvemorat ne coïncide pas toujours avec celui de la population d'âge scolaire. Dans certains gouvernorats, la disparité est nette : ainsi, le gouvemorat de Giza bénéficie d'un budget élevé proportionnellement à sa population, de même que les gouvernorats du Caire et de Beheira. En revanche, les dépenses d'enseignement dans les gouvernorats de Daqahliyya, Charqiyya, Assouan, Sinaï-nord et Minufiyya sont faibles par rapport à la population. Cette répartition est restée quasi inchangée entre 1982-83 et 1990-91.

20 L'inégalité profite généralement aux gouvernorats urbains (Le Caire et Giza en particulier), au détriment des gouvernorats ruraux (Haute-Égypte surtout), ceci alors que Le Caire et Giza, notamment, possèdent - comme nous l'avons vu plus haut-le taux minimum d'écoles primaires par rapport au nombre d'élèves potentiellement concernés; pour les écoles élémentaires et secondaires, la disparité est nettement moindre. Dans ces gouvernorats, par conséquent, les cycles élémentaire et secondaire sont favorisés par rapport à l'enseignement primaire.

\section{Répartition des dépenses d'enseignement entre frais courants et investissements}

21 Les parts de l'enseignement dans le produit intérieur brut et dans les dépenses budgétaires constituent des indices importants de la situation de ce secteur dans l'économie nationale. On peut noter la légère réduction du premier de ces deux indices dans les années 80 : il baisse de 2,2\% en 1980-81 à 2,0\% en 1989-90. En dépit de sa modestie par rapport aux pays industrialisés, où il s'élève à $5,2 \%$ environ, l'indice peut 
néanmoins être considéré comme positif. De même, la part réservée à l'enseignement pré-universitaire dans les dépenses budgétaires est passée de 5,1 en 1980-81 à 8,8 en 1989-90. Celle destinée à l'enseignement universitaire est montée de 2,5 en 1980-81 à 5,1 en 1989-90, indice a priori positif. A y regarder de près, ces données sont délicates à interpréter : il faut prendre en compte, d'une part, la place importante des salaires des enseignants dans les dépenses totales, d'autre part, la détérioration des salaires réels (près de $44 \%$ par rapport au niveau de 1980).

Tableau 3 : Dépenses d'enseignement 1980/1981 - 1989/1990 (en million LE) par niveau d'enseignement

\begin{tabular}{|c|c|c|c|c|c|c|c|c|c|c|c|c|}
\hline \multirow[b]{3}{*}{ Année } & \multicolumn{6}{|c|}{ Enseignement secondaire } & \multicolumn{6}{|c|}{ Enseignement universitaire } \\
\hline & \multirow[b]{2}{*}{ GSI* } & \multirow[b]{2}{*}{$\begin{array}{l}\text { GSI/ } \\
\text { SSE* }\end{array}$} & \multirow[b]{2}{*}{$\begin{array}{l}\text { Dépenses } \\
\text { nominales }\end{array}$} & \multicolumn{3}{|c|}{ Dépenses réelles } & \multirow[b]{2}{*}{ GSI* } & \multirow[b]{2}{*}{$\begin{array}{l}\text { GSI/ } \\
\text { SSE: }\end{array}$} & \multirow[b]{2}{*}{$\begin{array}{l}\text { Dépenses } \\
\text { nominales }\end{array}$} & \multicolumn{3}{|c|}{ Dépenses réelles } \\
\hline & & & & Salaires & $\begin{array}{l}\text { Entretien + } \\
\text { dépenses } \\
\text { courantes }\end{array}$ & Total & & & & Salaires & $\begin{array}{c}\text { Entretien + } \\
\text { dépenses } \\
\text { courantes }\end{array}$ & Total \\
\hline $\begin{array}{l}1980-81 \\
1983-84 \\
1986-87 \\
1989-90\end{array}$ & $\begin{array}{r}100 \\
162,0 \\
252,0 \\
431,5\end{array}$ & $\begin{array}{r}100 \\
97,5 \\
75,0 \\
66,0\end{array}$ & $\begin{array}{r}372,2 \\
774,0 \\
1172,4 \\
1788,8\end{array}$ & $\begin{array}{r}320,6 \\
709,8 \\
1444,9 \\
2538,5\end{array}$ & $\begin{array}{l}51,6 \\
50,6 \\
35,2 \\
26,3\end{array}$ & $\begin{array}{r}372,2 \\
760,4 \\
1480,1 \\
2564,8\end{array}$ & $\begin{array}{r}100 \\
162,0 \\
252,0 \\
431,5\end{array}$ & $\begin{array}{r}100 \\
97,5 \\
75,0 \\
66,0\end{array}$ & $\begin{array}{r}180,8 \\
418,0 \\
625,5 \\
1026,9\end{array}$ & $\begin{array}{r}100,7 \\
264,2 \\
558,5 \\
1078,2\end{array}$ & $\begin{array}{l}80,1 \\
99,0 \\
82,0 \\
73,1\end{array}$ & $\begin{array}{l}180,8 \\
363,2 \\
640,5 \\
1151,3\end{array}$ \\
\hline
\end{tabular}

GSI : Chiffre record pour les salaires gouvernementaux.

GSI/SSE : Pourcentage des salaires dans les dépenses du gouvernement

Source : World Bank, Poverty alleviation and adjustment in Egypt, Report n 8515. EGT, volume III : annexes, June 6,1990.

Pour obtenir une image plus fidèle de l'évolution réelle des dépenses d'enseignement, nous aurons recours à un indice qui tienne compte à la fois de révolution des salaires réels, de la part de ces derniers dans le budget et de l'indice des prix à la consommation (tableau 3).

23 Les dépenses d'équipements dans l'enseignement secondaire sont passées de 51,6 millions LE en 1980-81 à 26,3 millions LE en 1989-90, ce qui entraîne une diminution des achats de matériel indispensable au bon fonctionnement du service comme à l'entretien de l'infrastructure ${ }^{10}$.

24 Autre indice inquiétant dans ce domaine: la réduction de la part des dépenses d'investissement dans les dépenses totales, révélatrice d'une baisse des crédits attribués à l'investissement dans la création d'infrastructures (écoles, instituts et universités).

25 Par ailleurs, la part des salaires croît au détriment des dépenses courantes et des investissements (tableau 4), ce qui place le ministère de l'Education dans une situation délicate. En effet, la plus grosse partie des ressources du ministère est destinée aux salaires, au détriment de celles qui devraient être consacrées à l'entretien des infrastructures existantes ou à la création d'infrastructures nouvelles, cela sans que l'État puisse pour autant offrir aux enseignants un niveau de salaire suffisamment motivant.

26 De ce fait, l'État a été amené à imposer, dans tous les cycles de l'enseignement, une certaine austérité pour compenser la diminution de la part du budget consacrée aux dépenses non salariales, mesure qui a eu des répercussions négatives sur l'égalité des chances, surtout dans le primaire. 
Tableau 4 : Répartition proportionnelle des dépenses consacrées à l'enseignement en Égypte (\%)

\begin{tabular}{|c|c|c|c|c|c|}
\hline Année & Salaires & $\begin{array}{c}\text { Dépenses } \\
\text { couranles }\end{array}$ & Investiss. & $\begin{array}{c}\text { Transfert } \\
\text { de capitaux }\end{array}$ & Total \\
\hline $1982-83$ & 64,2 & 16,3 & 16,8 & 2,7 & 100,0 \\
$1985-86$ & 70,4 & 15,3 & 12,3 & 2,0 & 100,0 \\
$1988-89$ & 72,8 & 11,9 & 13,9 & 1,4 & 100,0 \\
$1990-91$ & 72,8 & 13,1 & 13,6 & 0,6 & 100,0 \\
\hline
\end{tabular}

Source : calculs effectués à partir des données du ministère des Finances

27 Les indices s'accumulent du caractère prohibitif des frais d'éducation pour les familles défavorisées, ce qui se traduit par un taux de non scolarisation particulièrement élevé dans les quartiers pauvres. Aux frais irréductibles, qui varient actuellement entre 20 et 30 LE par élève du cycle primaire, il faut ajouter les 3 à 5 LE par matière et par mois que coûtent les cours de rattrapage ${ }^{11}$; encore faut-il préciser que le ministère de l'Education vient de codifier ces cours en les intégrant à l'école publique, ceci pour lutter contre le phénomène des leçons particulières données hors de l'école. Désormais, l'école organise ces cours de rattrapage moyennant une somme fixe. Le nombre d'écoliers inscrits à ces cours atteint quasiment la moitié de l'effectif inscrit.

En conséquence, le taux de non scolarisation a augmenté. Pour le cycle primaire, il a atteint un pourcentage variant entre 19,4 et 23 pour la période allant de 1963-64 à 1969-70 $0^{12}$, et jusqu'à 30\% dans la période allant de 1978 à $1985^{13}$. En réalité, le taux de non inscrits est un des aspects des "privilèges de classe" favorisés par le système d'enseignement actuel: certaines études effectuées dans ce domaine indiquent que c'est parmi les catégories défavorisées, plutôt que parmi celles à revenu élevé, que l'on constate une élévation du taux de non inscrits ${ }^{14}$.

Autre conséquence de l'augmentation de la masse salariale au détriment des dépenses courantes et d'investissement - et autre facteur d'inégalité des chances -: la surpopulation scolaire, c'est-à-dire l'augmentation de la densité d'élèves par classe pour les cycles primaire et élémentaire. La moyenne nationale actuelle pour le primaire est d'environ 45 élèves par classe et atteint parfois $60^{15}$, situation qui rend quasiment impossible tout enseignement. Le niveau d'assimilation des écoliers s'affaiblit et le recours aux leçons particulières devient inévitable pour réussir.

\section{Répartition des dépenses d'enseignement entre les divers cycles}

Les données en la matière indiquent une répartition avantageuse pour les cycles secondaire et supérieur par rapport à leur effectif, et désavantageuse pour le cycle primaire, ce qui, en fait, avantage les catégories à revenu moyen et élevé. Les études entreprises dans ce domaine montrent clairement que l'extension de l'enseignement supérieur subventionné par l'État consolide la structure sociale actuelle: une telle subvention constitue en fait un transfert de revenu dés pauvres vers la classe moyenne ${ }^{16}$.

31 Selon une étude récente de la Banque Mondiale $^{17}$, les individus qui parviennent au niveau universitaire sont subventionnés par le reste de la société : $70 \%$ de la population est en effet constituée de familles dont les enfants ne sont pas scolarisés ou n'ont pas 
dépassé le cycle primaire, alors qu'ils ne représentent que $20 \%$ des dépenses d'enseignement. En revanche, les étudiants ayant accès à l'université, qui représentent $10 \%$ de leur tranche d'âge, bénéficient de $50 \%$ du total de ces dépenses. Les données pour 1987-88 montrent la persistance de ce mode de répartition, qui favorise l'enseignement secondaire et supérieur au détriment de l'enseignement primaire (tableau 5).

Tableau 5 : Répartition proportionnelle des ressources entre les différents types et cycles d'enseignement 1987/1988 (\%)

\begin{tabular}{|l|r|r|r|r|}
\hline \multicolumn{1}{|c|}{ Cycle } & Investiss. & $\begin{array}{c}\text { Dépenses } \\
\text { courantes }\end{array}$ & Total & Elèves \\
\hline Primaire & 31,5 & 35,0 & 34,7 & 59,0 \\
Préparatoire & 11,8 & 16,7 & 16,0 & 21,8 \\
Secondaire & 2,2 & 4,8 & 4,5 & 5,0 \\
Second. technique & 10,8 & 11,5 & 11,5 & 8,0 \\
Instituts supérieurs & 1,5 & 1,2 & 1,2 & 1,7 \\
Universités & 42,2 & 30,8 & 32,1 & 4,5 \\
Total & 100,0 & 100,0 & 100,0 & 100,0 \\
\hline
\end{tabular}

Source : World Bank, Poverty alleviation and adjustment in Egypt, Report no. 8515 EGT, volume III M Annexes, June 61990, annexe J, table J3, p. 59.

L'écart est manifeste : les élèves du cycle primaire, qui représentent $59 \%$ des effectifs, obtiennent moins de $35 \%$ des dépenses d'enseignement, alors que ceux du cycle supérieur, qui n'en représentent que 4,5\%, consomment 32,1\% du budget. En revanche, les effectifs des cycles préparatoire, secondaire général, secondaire technique et des instituts supérieurs sont proportionnels aux dépenses d'enseignement qui leurs sont attribuées.

33 Selon les études effectuées dans divers pays ${ }^{18}$, le taux des subventions accordées par l'État à l'enseignement croît en fonction de la hiérarchie des divers cycles. De même, le rendement de ces subventions ira en décroissant pour les catégories les plus défavorisées, le niveau d'enseignement étant ainsi fonction du niveau de revenus de la catégorie à laquelle appartient l'élève. Les études effectuées en Égypte à cet égard confirment que l'écart existant dans la répartition des dépenses d'enseignement favorise les catégories à revenu élevé. En fait, 80 \% des élèves de condition modeste ne parviendront jamais au cycle supérieur, car ils n'obtiennent pas le nombre de points requis pour y être admis. En revanche, parmi les étudiants des facultés où l'admission nécessite une note élevée, une large proportion appartient à la tranche supérieure de la classe moyenne dans les zones urbaines ${ }^{19}$. Ainsi, à l'université du Caire, plus de $85 \%$ des étudiants sont issus de la petite bourgeoisie (professions libérales, cadres supérieurs). Les enfants d'ouvriers et d'agriculteurs n'en constituent qu'un dixième ${ }^{20}$.

Notons encore que cette orientation du mode de dépenses est due à une insuffisance des ressources disponibles qui se traduit par une qualité inférieure de l'enseignement public. Ainsi les enfants des classes moyennes et élevées seront-ils relativement privilégiés, la tendance croissante consistant à les inscrire dans des établissements privés, de meilleur niveau, et à recourir aux leçons particulières ${ }^{21}$. Cette situation se 
maintient depuis le début des années 80 : le taux de dépenses consacrées à l'enseignement supérieur est de $30 \%$ au moins du budget, du début à la fin du cycle.

Cet état de choses s'est répercuté sur le coût de scolarisation pré-universitaire et universitaire. En 1980-81, ce coût représentait, par élève de l'enseignement préuniversitaire, 51,48 LE, soit 15,5\% du total du coût représenté par un étudiant de l'enseignement supérieur pour la même période (331,06 LE). En 1989-90, la scolarisation d'un élève de l'enseignement pré-universitaire coûte $230,66 \mathrm{LE}$, soit $11,8 \%$ seulement du coût de scolarisation d'un étudiant de l'enseignement supérieur pour la même période $(1.951,36 \mathrm{LE})$ selon les prix constants de 1980. Autrement dit, le coût de scolarisation d'un élève de niveau pré-universitaire a baissé durant les années 80 (en dépit de sa réduction partielle absolue et relative), par rapport au coût de scolarisation d'un élève du cycle supérieur. C'est là la conséquence de l'augmentation du coût de scolarisation d'un étudiant de l'enseignement supérieur dans une proportion supérieure $(489 \%)$ à celle du coût de scolarisation d'un élève de l'enseignement préuniversitaire (348 \%) pour la même période (tableau 6).

Tableau 6 : Coûts de l'enseignement général et supérieur aux prix fixes de l'année 1980 (en million LE)

\begin{tabular}{|c|c|c|c|c|}
\hline Année & $\begin{array}{c}(1) \\
\text { Enseignement } \\
\text { général }\end{array}$ & $\begin{array}{c}(2) \\
\text { Enseignement } \\
\text { supérieur }\end{array}$ & $\begin{array}{c}(3) \\
\text { Total }\end{array}$ & $\begin{array}{c}\text { Rapport de } \\
(2) \text { à (3) } \\
\%\end{array}$ \\
\hline $1980-81$ & 372,2 & 180,8 & 553,0 & 32,7 \\
$1983-84$ & 760,4 & 363,2 & 1122,6 & 32,3 \\
$1986-87$ & 1480,1 & 640,5 & 2120,6 & 30,2 \\
$1989-90$ & 2564,8 & 1151,3 & 3716,1 & 30,9 \\
\hline
\end{tabular}

Source : calculs effectués à partir des données du tableau 3.

\section{Relations enseignement privé/enseignement public}

On peut encore mesurer le degré d'égalité des chances en matière d'enseignement en examinant le rapport enseignement privé/enseignement public, les établissements privés payants attirant généralement les enfants de familles à revenu élevé, et les établissements publics subventionnés ou gratuits, les enfants des familles à faibles revenus (tableau 7 infra p.190).

Non seulement l'extension de l'enseignement obligatoire et gratuit, qui visait à réaliser l'égalité des chances, ne s'est pas accompagnée d'une réduction du rapport enseignement privé/enseignement public, mais l'inverse s'est même produit: tandis que le nombre d'établissements du cycle primaire passait de 303 (5.267 classes) en 1976-77, à 814 (8.699 classes) en 1989-90, la proportion des écoles privées par rapport à l'ensemble des écoles primaires passait de $3 \%$ en 1976-77 à 5,8 \% en 1989-90 (et les classes de 5,2 \% à $6,5 \%$ dans la même période).

Un accroissement du taux d'écoles privées pourrait contribuer à réaliser une certaine équité, les catégories à revenus élevés payant la scolarisation de leurs enfants. Mais cette dualité du système d'enseignement égyptien aurait, à long terme, un impact négatif. Avec la détérioration continue de la qualité de l'enseignement public, 
conséquence d'une extension non étayée par des moyens financiers suffisants, les classes privilégiées obtiennent un enseignement de qualité leur permettant d'accéder aux meilleurs emplois, tandis que la médiocrité des services fournis aux classes défavorisées ne leur permet de gravir ni les échelons de l'enseignement ni, plus tard, ceux de l'emploi.

Cette situation se traduit par une très faible évolution du niveau éducatif des citoyens : en trente ans (1947-1986), le pourcentage d'Égyptiens ayant atteint un niveau universitaire ou post-universitaire n'est passé que de $0,4 \%$ à $3,1 \%$ du total des effectifs scolarisés (tableaux 8, 9 \&.10 infra pp. 190-191).

\section{L'inégalité des chances face a la scolarisation}

Les déterminants de la demande individuelle d'enseignement sont multiplespolitiques, sociaux, économiques... Mais c'est le rendement de l'enseignement, anticipé par les individus, qui en détermine la demande. Par conséquent, les facteurs essentiels intervenant dans la demande individuelle d'enseignement seraient, d'une part, la situation du marché du travail, d'autre part, la structure du coût individuel dans le système d'enseignement ${ }^{22}$.

41 Ce document se contentera d'aborder le coût individuel direct de l'enseignement en Égypte (calculé d'après les dépenses d'enseignement indiquées dans les études sur le budget familial), élément considéré comme un des plus déterminants -sinon le plus déterminant- de la demande individuelle d'enseignement.

D'une manière générale, ce coût a augmenté entre 1974-75 et 1990-91. Remarquons que cette augmentation a provoqué, chez les catégories défavorisées de milieu urbain ou rural, une augmentation de la part des dépenses d'enseignement dans l'ensemble des dépenses; en revanche, ce taux est demeuré sensiblement le même pour les catégories à moyens et hauts revenus. Cette tendance - la majoration du coût de l'enseignement pour les familles démunies - s'est confirmée pendant la période 1981-82 à 1990-91, et cela plus nettement que pendant la période 1974-75 à 1981-82. Elle a frappé davantage les zones rurales que les zones urbaines au cours de la période la plus récente (tableau $11)$.

Tableau 7 : Volume de l'enseignement privé par rapport à l'enseignement public 1976-77 et 1989-90

\begin{tabular}{|c|c|c|c|c|c|c|c|c|c|}
\hline & \multicolumn{3}{|c|}{ Enseignement primairध } & \multicolumn{3}{|c|}{ Enseignement préparatoire } & \multicolumn{3}{|c|}{ Enseignement secondaire } \\
\hline & $\begin{array}{l}\text { Public } \\
\text { No. }\end{array}$ & $\begin{array}{l}\text { Privê } \\
\text { Nb. }\end{array}$ & $\begin{array}{c}\% \\
\text { privé/tot. }\end{array}$ & $\begin{array}{l}\text { Public } \\
\text { Nb. }\end{array}$ & $\begin{array}{l}\text { Privé } \\
\text { Nb. }\end{array}$ & $\begin{array}{c}\% \\
\text { privé/tot. }\end{array}$ & $\begin{array}{l}\text { Public } \\
\text { Nb. }\end{array}$ & $\begin{array}{l}\text { Prive } \\
\text { No. }\end{array}$ & $\begin{array}{c}\% \\
\text { prive } / \text { tot. }\end{array}$ \\
\hline $\begin{array}{l}1976.77 \\
\text { Nb. d'écoles } \\
\text { Nb. de classes } \\
\text { 1999.90 } \\
\text { Nb. docoles } \\
\text { Nb. de classes }\end{array}$ & $\begin{array}{r}9.697 \\
96.368 \\
\\
13.953 \\
133.418\end{array}$ & $\begin{array}{r}303 \\
5.267 \\
\\
\\
814 \\
8.699\end{array}$ & $\begin{array}{l}3,0 \% \\
5,2 \%\end{array}$ & $\begin{array}{r}1.578 \\
28.141\end{array}$ & $\begin{array}{r}242 \\
7.747\end{array}$ & $\begin{array}{r}13,8 \% \\
21,6 \% \\
\\
6,9 \% \\
\end{array}$ & $\begin{array}{r}323 \\
7.718\end{array}$ & $\begin{array}{r}121 \\
2.138\end{array}$ & $\begin{array}{l}27,2 \% \\
21,7 \%\end{array}$ \\
\hline
\end{tabular}

Source : 1976-77 : M. 'Abd al-Fadil (1982), table 11.4, p. 365. 1989-90 : données du ministère de l'Éducation. 
Tableau 8 : Répartition de la population selon le niveau d'éducation 1947-1986 (périodes de 10 ans) en milliers

\begin{tabular}{|c|c|c|c|c|c|c|c|c|c|c|}
\hline \multirow{2}{*}{ Niveau d'éducation } & \multicolumn{2}{|c|}{1947} & \multicolumn{2}{|c|}{1960} & \multicolumn{2}{|c|}{1966} & \multicolumn{2}{|c|}{1976} & \multicolumn{2}{|c|}{1986} \\
\hline & No. & $\%$ & Nb. & $\%$ & No. & $\%$ & Nab. & $\%$ & $\mathrm{Na}$ & $\%$ \\
\hline Analohabète & 10.900 & 78,0 & 12.726 & 70,5 & 13.770 & 65,3 & 15.611 & 56,5 & 17.288 & 50,0 \\
\hline Lit et écrit & 2.911 & 20,8 & 4.356 & 24,2 & 5.886 & 27,9 & 6.923 & 25,1 & 9.266 & 26,9 \\
\hline Dipłome secondaire ou plus & 113 & 0,8 & 801 & 4,4 & 1.193 & 5.7 & 4.475 & 16,2 & 6.932 & 20,0 \\
\hline Diplome universitaire ou plus & 57 & 0,4 & 170 & 0,9 & 235 & 1,1 & 606 & 2,2 & 1.064 & 3.1 \\
\hline Total & 13,981 & 100,00 & 18.053 & 100,00 & 21.084 & 100,00 & 27,615 & 100,00 & 34.550 & 100,00 \\
\hline
\end{tabular}

Source : Années 1947, 1960, 1966, 1976 : Sa'd al-Din Ibrahim, « Social mobility and income distribution in Égypt 1952-1977 » in Gouda 'Abd al-Khaliq and Robert Tignor (éd.), The political economy of income distribution in Egypt, Holmes \& Meger Publishers, Inc., New-York, 1982, table 12-11, p. 402. Année 1986 : CAPMAS, Annuaire statistique, 1992, République Arabe d'Égypte, juin 1992, tableaux 1-13, p. 34.

Tableau 9 : Évolution proportionnelle du niveau d'éducation 1947-1986

\begin{tabular}{|c|c|c|c|c|c|c|c|c|}
\hline \multirow[b]{2}{*}{ Niveau d'éducation } & \multicolumn{2}{|c|}{$1947 \cdot 1960$} & \multicolumn{2}{|c|}{$1960-1966$} & \multicolumn{2}{|c|}{$1966-1976$} & \multicolumn{2}{|c|}{$1976-1986$} \\
\hline & $\begin{array}{c}\text { Evolution } \\
\text { sur la } \\
\text { période }\end{array}$ & $\begin{array}{c}\text { Taux } \\
\text { annued } \\
\text { d'évolut. }\end{array}$ & $\begin{array}{c}\text { Evolution } \\
\text { sur la } \\
\text { période }\end{array}$ & $\begin{array}{c}\text { Taux } \\
\text { annuet } \\
\text { c'évolut. }\end{array}$ & $\begin{array}{c}\text { Evolution } \\
\text { sur la } \\
\text { periode }\end{array}$ & $\begin{array}{c}\text { Taux } \\
\text { annuel } \\
\text { d'évolut. }\end{array}$ & $\begin{array}{c}\text { Evolution } \\
\text { sur la } \\
\text { période }\end{array}$ & $\begin{array}{c}\text { Taux } \\
\text { annued } \\
\text { d'évolut. }\end{array}$ \\
\hline Analphabète & 16,8 & 1,3 & 8,2 & 1,4 & 13,4 & 1,3 & 10,7 & 1,07 \\
\hline Lit et écrit & 49,6 & 3,8 & 35,1 & 5,6 & 17,6 & 1,8 & 33,8 & 3,38 \\
\hline Diplòme secondaire ou pius & 608,8 & 46,8 & 48,9 & 8,2 & 275.1 & 27,5 & 111,3 & 11,13 \\
\hline Diplome universitaire ou plus & 198,2 & 15,2 & 38,2 & 6,4 & 157,9 & 15,8 & 75,5 & 7,55 \\
\hline Total & 29,2 & 2,0 & 16,8 & 2,6 & 31,0 & 2,7 & 25,1 & 2,51 \\
\hline
\end{tabular}

Source : calculs effectuées à partir du tableau 10.

Tableau 10 : Évolution de la moyenne des dépenses familiales consacrées à l'enseignement 1974-75, 1981-82, 1991-92

\begin{tabular}{|c|c|c|c|c|c|c|}
\hline & \multicolumn{3}{|c|}{$\begin{array}{l}\text { Moyenne des dépenses famidiales } \\
\text { dans les classes défavorisées }\end{array}$} & \multicolumn{3}{|c|}{$\begin{array}{l}\text { Moyenne des dépenses familiales } \\
\text { dans les classes moyenne et aisée }\end{array}$} \\
\hline & $74 / 75$ & $81 / 82$ & $91 / 92$ & $74 / 75$ & $81 / 82$ & $91 / 92$ \\
\hline $\begin{array}{l}\text { Régions urbaines } \\
\text { (1) Dépenses pour l'enseignement } \\
\text { (2) Total des dépenses annutelles } \\
\text { Pourcentage (1) / (2) } \\
\text { Réglons rurales } \\
\text { (1) Dépenses pour l'enseignement } \\
\text { (2) Total de dépenses annuelles } \\
\text { Pourcentage (1) / (2) }\end{array}$ & $\begin{array}{r}2,09 \\
215,05 \\
0,90\end{array}$ & $\begin{array}{r}7,50 \\
744,60 \\
1,00 \\
\\
\\
2,44 \\
547.10 \\
0.44\end{array}$ & $\begin{array}{r}51,0 \\
2664,6 \\
1,90\end{array}$ & $\begin{array}{r}39,70 \\
1280,50 \\
3,10\end{array}$ & $\begin{array}{r}92,20 \\
4719,00 \\
1,95 \\
\\
27,50 \\
3715,30 \\
0,74\end{array}$ & $\begin{array}{r}339,20 \\
11224,30 \\
3,00\end{array}$ \\
\hline
\end{tabular}

Source : CAPMAS.

43 On peut expliquer cette situation par l'ampleur du recours aux leçons particulières, conséquence de la détérioration de la qualité de l'enseignement public, ainsi que par le recours aux manuels extra-scolaires, populaires parmi les élèves et exigés des enseignants pour leurs leçons particulières en raison de la médiocrité des livres scolaires. Autant de facteurs qui ont augmenté le coût de l'enseignement pour les plus démunis et qui risquent de faire décroître la demande d'enseignement, tandis que le coût de l'enseignement a été réduit ou stabilisé pour les classes moyennes ou aisées. Il 
en résulte, avec le temps, une inégalité croissante des chances d'accès à l'enseignement, notamment depuis les quinze dernières années (1974-75 à 1990-91).

Tableau 11 : Augmentation du coût individuel direct de l'enseignement en Égypte

\begin{tabular}{|c|c|c|}
\hline $\begin{array}{c}\text { Régions } \\
\text { urbaine / rurale }\end{array}$ & $\begin{array}{c}81 / 82 \\
\text { par rap. à } \\
74 / 75\end{array}$ & $\begin{array}{c}90 / 91 \\
\text { par rap. à } \\
81 / 82\end{array}$ \\
\hline $\begin{array}{l}\text { Régions urbaines } \\
\text { Taux d'augmentation des prix “** } \\
\text { Tx daugment. du coût individuel de l'enseignement * } \\
\text { Tx daugment. du total des dépenses annuelles * } \\
\text { Tx d'augment. du coût individuel de l'enseignement ..- } \\
\text { Tx d'augment. du tolal des dépenses annuelles "* }\end{array}$ & $\begin{array}{r}55,00 \% \\
275,00 \% \\
246,00 \% \\
132,20 \% \\
268,60 \%\end{array}$ & $\begin{array}{l}347,70 \% \\
580,00 \% \\
357,80 \% \\
267,80 \% \\
137,80 \%\end{array}$ \\
\hline $\begin{array}{l}\text { Réglons rurales } \\
\text { Tx d'augment. des prix .." } \\
\text { Tx daugment. du coût individuel de renseignement " } \\
\text { Tx d'augment. du lotal des dépenses annuelles " } \\
\text { Tx d'augment. du coúl individuel de renseignement ** }\end{array}$ & $\begin{array}{r}57,70 \% \\
248,50 \% \\
233,60 \% \\
110,00 \% \\
271,10 \%\end{array}$ & $\begin{array}{r}339,60 \% \\
1025,00 \% \\
321,60 \% \\
536,30 \% \\
170,50 \%\end{array}$ \\
\hline
\end{tabular}

Source : Banque Nationale d'Égypte.

*Classes défavorisées.

**Classes moyenne et aisée.

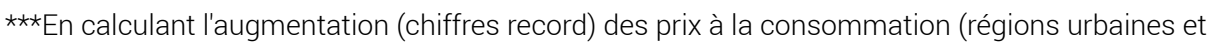
rurales).

\section{Conclusion}

Nous évoquerons pour conclure les effets prévisibles du programme de redressement économique sur l'enseignement et les chances de scolarisation.

\section{Effets à court terme}

45 1) Les politiques financières prévues par le programme de redressement vont très probablement se répercuter sur l'offre de l'enseignement officiel. Réduire le déficit budgétaire (en comptant la réduction des dépenses, de près de $51,1 \%$ en 1990-91 à environ $44 \%$ en 1995-96), c'est aboutir à une réduction du volume des subventions destinées à l'enseignement public, d'où une diminution quantitative et qualitative de l'enseignement et une réduction des services non pédagogiques liés à l'enseignement (cantine, services sanitaires, etc.).

46 On peut par conséquent s'attendre à un effet négatif sur le volume de l'enseignement (déjà fortement réduit si l'on en croit les taux de scolarisation, lesquels n'expriment pourtant pas la situation réelle de l'enseignement en Égypte si l'on considère le pourcentage élevé de non scolarisation - environ $30 \%$ ). Ajoutons à cela le quota élevé 
que représentent les salaires par rapport au budget total du ministère de l'Education ( $94 \%)$, et le quota de dépenses par élève en équipements aux prix stabilisés de la fin des années 80 , qui a été réduit au cinquième de son montant par rapport à ce qu'il était au début de la décennie. Une plus grande réduction des dépenses dans ce domaine risque d'entraîner une détérioration supplémentaire de l'enseignement, quantitative et qualitative.

De même, une réduction des dépenses d'investissement dans l'enseignement (conséquence de la réduction des investissements en général, sans compter le montant déjà minimal des ressources allouées à ce poste et la montée des prix des terrains en zone urbaine), risque de freiner la construction de nouveaux bâtiments scolaires. D'où, là encore, une stagnation de l'enseignement en volume et en qualité du fait de la surcharge des classes.

Quant à la stabilisation du budget par la réduction du nombre d'enseignants et la baisse des salaires réels, elle ne peut elle aussi qu'influer négativement sur ce secteur. Les catégories les plus touchées seront certainement les plus démunies, que ce soit en milieu urbain ou en milieu rural : ces catégories sont moins aptes à se protéger de ces effets négatifs que les catégories plus à revenus élevés ${ }^{23}$, et ce sont elles qui bénéficient en premier lieu de l'enseignement officiel. Elles vont donc pâtir de tout ce qui en amoindrira le volume et la qualité. Les gouvernorats qui souffrent le plus de la réduction du volume de l'enseignement et du budget (ceux de Haute-Égypte) ne peuvent guère espérer une amélioration des services éducatifs dans ces circonstances. Ainsi l'écart entre gouvernorats va-t-il persister, voire s'accroître.

2) Les politiques financières prévues par le programme de redressement économique peuvent également se répercuter sur la demande individuelle d'enseignement. Leur effet majeur à court terme ${ }^{24}$ sera une baisse de la demande par suite d'une réduction anticipée du revenu, sans compter qu'une majoration des frais d'inscription scolaire est prévue. La demande d'enseignement va donc suivre la baisse de revenus des familles concernées : il leur faudra peut-être, tôt ou tard, décider d'interrompre la scolarité de leurs enfants, ce qui, inexorablement, accentuerait l'inégalité des chances, d'autant que la diminution des revenus, ajoutée à la hausse des prix des services de santé et des denrées alimentaires, ne peut qu'entraîner un amoindrissement du niveau de vie sur le plan sanitaire et alimentaire chez les familles démunies, et réduire par conséquent leurs facultés d'assimilation. La demande d'enseignement peut encore décroître si le temps normalement consacré à l'enseignement se reporte sur d'autres activités, économiques ou ménagères, en particulier pour les filles dans le monde rural ${ }^{25}$. Toutefois, l'inverse pourrait se produire si les possibilités d'emploi des enfants diminuaient.

\section{Effets à long terme}

50 Ils reposent dans une large mesure sur le succès du programme de redressement, lequel peut accroitre le rendement de tous les types d'enseignement et les ressources consacrées à ce secteur. Mais simultanément, le revenu du travail de l'enfant restera supérieur au revenu anticipé de sa scolarisation ${ }^{26}$.

51 Il existe un antagonisme fondamental dans le programme de redressement proposé : il vise en effet à mettre en valeur l'élément travail, considéré comme essentiel et, de plus, relativement avantageux pour l'Égypte. Mais cela implique d'élever le niveau 
d'aptitude des individus en élevant, d'une part, celui de l'enseignement et de la formation et, d'autre part, le niveau de vie (sur les plans sanitaire et alimentaire) des salariés. D'où une majoration indispensable de la part de budget consacrée aux services sociaux... et non sa réduction, comme le préconise le programme de redressement.

Le séisme qui a ébranlé l'Égypte le 12 octobre 1992 a gravement touché le secteur de l'enseignement : 1.087 écoles se sont totalement effondrées, 2.301 se sont en partie écroulées, 3.569 doivent être restaurées. N'y a-t-il pas là la preuve qu'il faut reconsidérer le programme de réforme économique et la réduction des dépenses qu'il implique dans ce secteur? Tout ce qui aura pour l'enseignement des effets négatifs risque de compromettre le succès de la réforme en se retournant contre elle.

\section{NOTES}

1. World Bank, Poverty Alleviation and Adjustment in Egypt, Report $n^{\circ} 8515-\mathrm{EGT}$, volume II ; Main Report, June 6,1990, p.76.

2. IBRAHIM Saad al-Din, "Social mobility and income distribution in Egypt, 1952-1972 ", in The political economy of income distribution in Egypt, Holmes and Meyers Publishers, Inc., New-York, 1982, p375-434.

3. ABD AL-PADIL Mahmoud, «Educational expansion and income distribution in Égypt, 1952-1977 », in The political economy..., op. cit., p.354.

4. W. B., Poverty Alleviation..., op. cit., p.74.

5. Ibid, p.76.

6. PSACHAROPOULOS G. and WOODHALL M., L'Éducation, p.269.

7. Central administration for public mobilization and statistics (CAPMAS), Recensement général 1986, Énumération globale, Caractéristiques de la population, vol.2, résultats finaux pour tout le territoire, Le Caire, 1990.

8. Ibid.

9. Du fait de l'absence de la tranche d'âge (12-18 ans) dans la classification du recensement général de la population, 1986.

10. W.B., Poverty Alleviation..., op. cit., p.116.

11. Ibid, p.117-118.

12. ABD AL-FADIL, op. cit., p.355.

13. SULAYMAN Salwa et al., Le droit au travail dans l'économie égyptienne, Centre national de recherches sociales et criminologiques (CNRSC), Le Caire, 1987.

14. ABD AL-FADIL, op. cit., p.353.

15. W.B., Poverty Alleviation..., op. cit., p.118.

16. KAMEL Samia Mustafa, Faisabilité économique de l'enseignement supérieur en Égypte, Thèse de doctorat (non publiée), Faculté d'économie et de sciences politiques, Université du Caire, 1990, p. 199.

17. W.B., Poverty Alleviation..., op. cit., p.77-78.

18. ABD AL-FADIL, op. cit., p.361.

19. Ibid., p.365-367.

20. AL-AYYUBI Nazih Nassib, La politique de l'enseignement en Égypte, Etude politique et administrative, Centre d'Etudes politiques et stratégiques, Le Caire, 1978. 
21. ABD AL-FADIL, op. cit., p.367-371.

22. KAMEL Samia Mustafa, op. cit., p.149.

23. W.B., Poverty Alleviation..., op. cit., p.105.

24. Ibid, p.106.

25. Ibid, p. 106.

26. Idem.

INDEX

Mots-clés : économie, enseignement, réforme économique, scolarisation, enseignement féminin

\section{AUTEUR}

MONA MUSTAFA AL-BARADI'I

Université du Caire 